\title{
Is neutrophil the dominant IL-17 producer in psoriasis?
}

\author{
Kento Mizutani, Yoshiaki Matsushima, Karin Okada, Akisa Yamagiwa, Tomoko Akeda, Makoto Kondo, \\ Masato Kakeda, Koji Habe, Keiichi Yamanaka \\ Department of Dermatology, Mie University, Graduate School of Medicine, Mie, Japan
}

(a) Normal

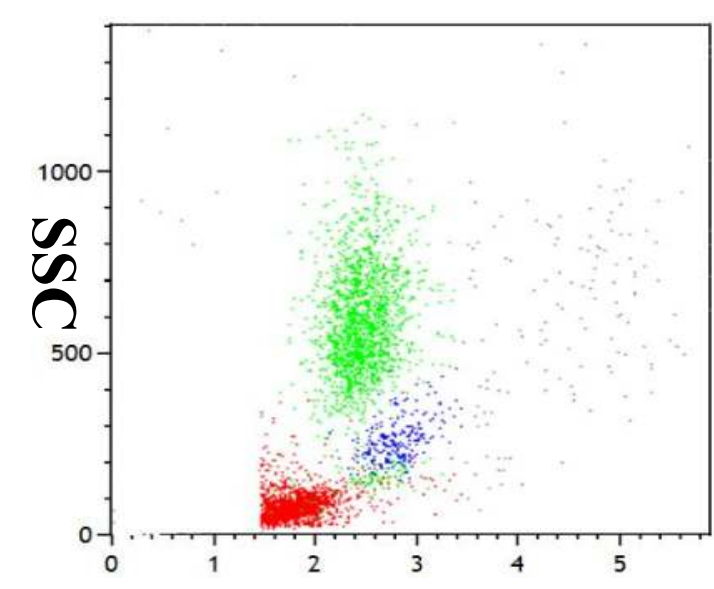

FSC
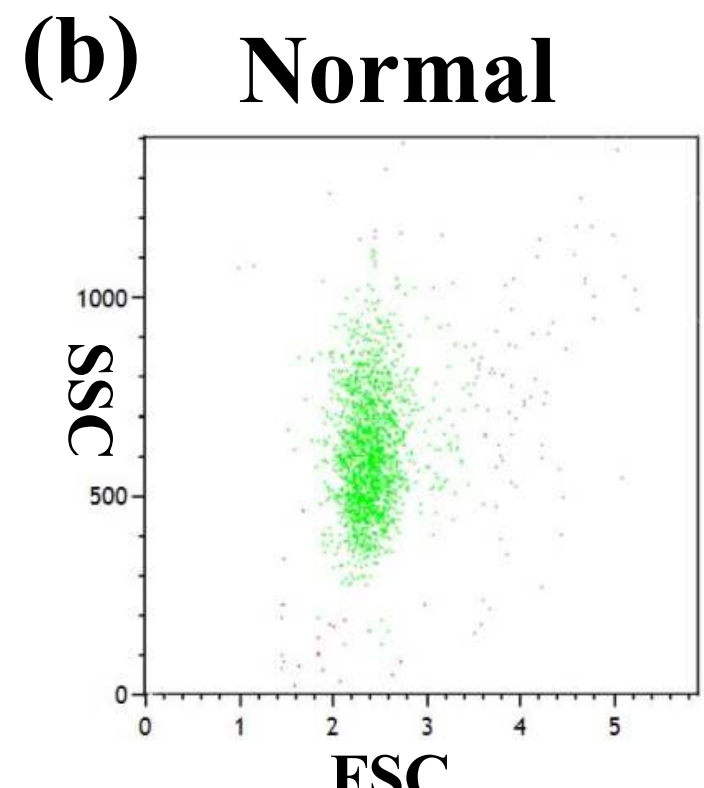

FSC
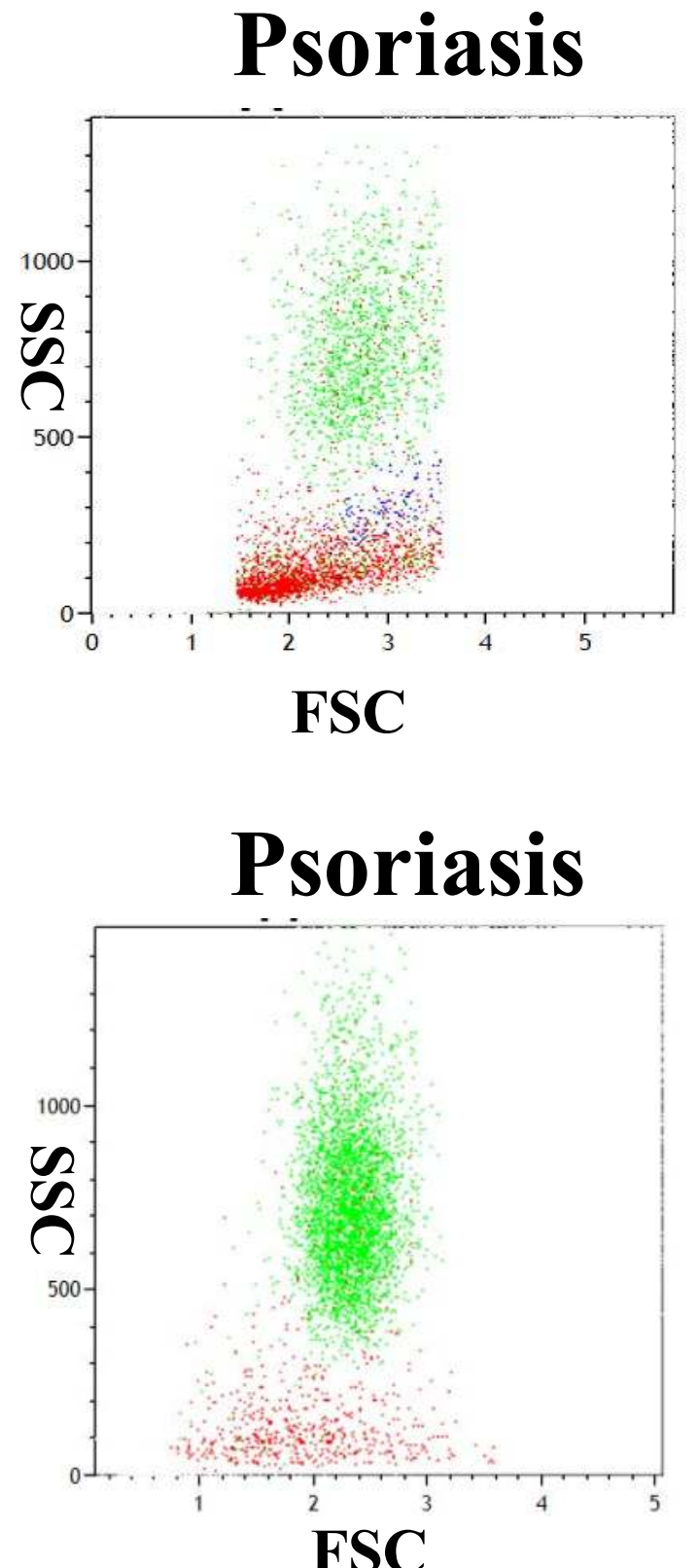

(c)

IL-17A

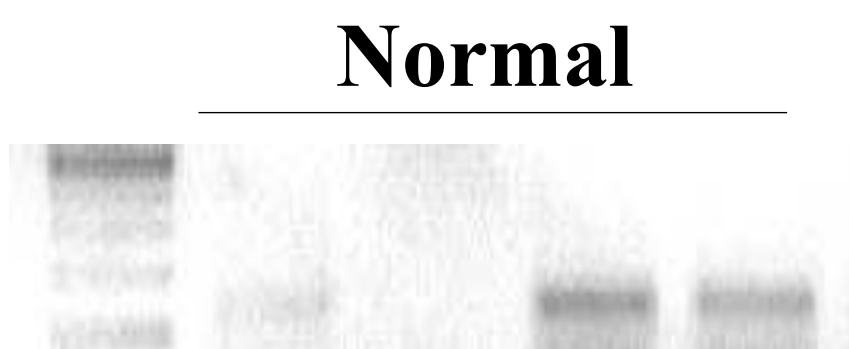

Psoriasis

GAPDH
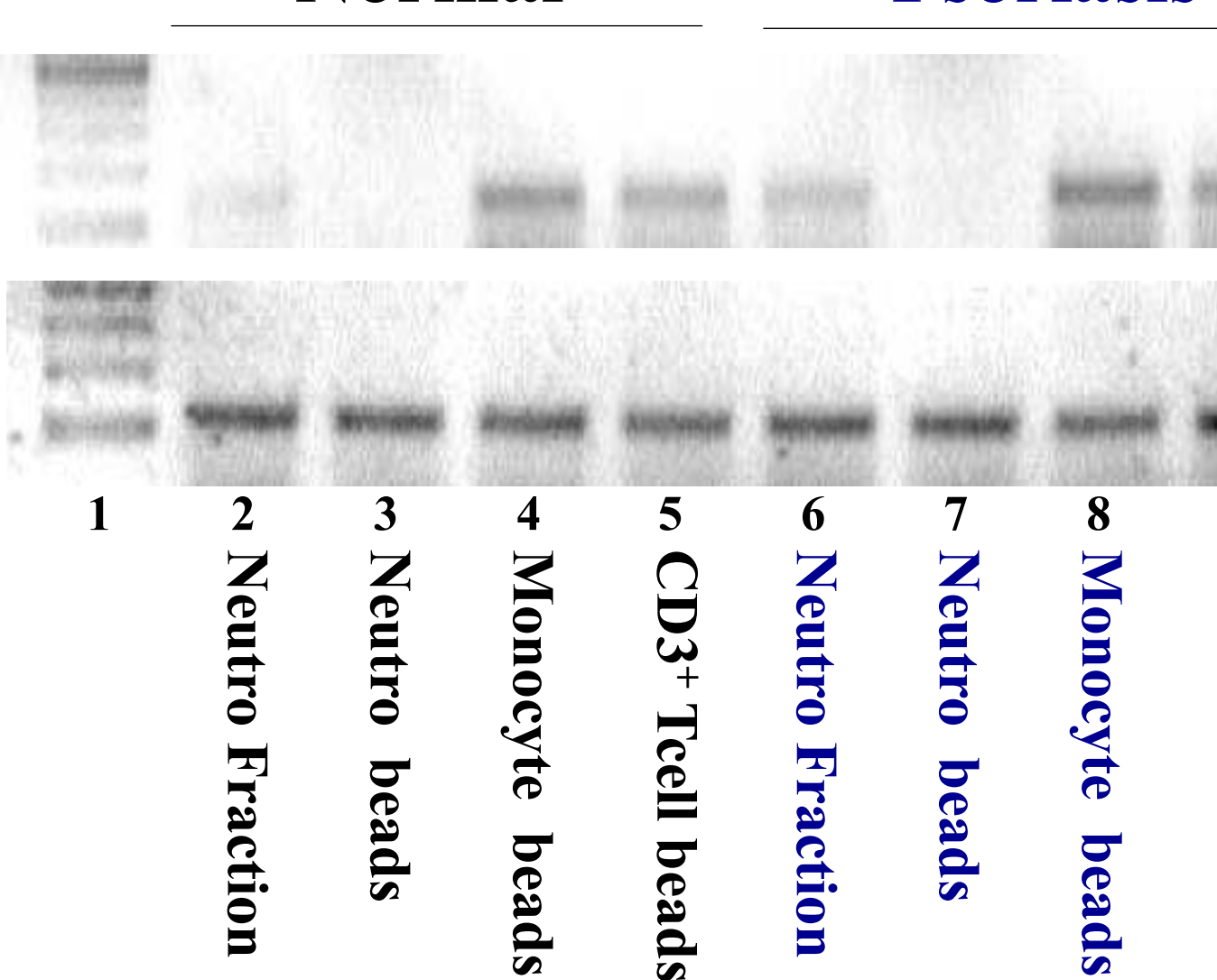

Figure 1. (a) Neutrophils purified by density gradient centrifugation were analyzed by flow cytometry (monocyte is $\mathrm{CD} 14^{+}$high; blue, neutrophil is $\mathrm{CD} 14^{+}$low; green, $\mathrm{CD}^{+} \mathrm{T}$ cell is red). (b) The purity of the neutrophils collected by a specific magnetic beads separation shows remarkable improvement in samples from normal control and severe psoriasis patient. (c) mRNA of IL-17A and GAPDH was amplified by RT-PCR. Lanes 1 and 10 were size markers. FSC, forward scatter; SSC, side scatter.

\section{Introduction}

- Neutrophil is the majority in the psoriatic lesions, and have been suggested IL-17 producer, however, the real IL-17 supplier in psoriasis is still controversial.

- Presence of IL-17 immuno-reactive neutrophils in the psoriatic skin lesions has been reported histopathologically as well as in peripheral blood by flow cytometric analysis. ${ }^{1}$

- A report declared absence of IL-17 mRNA expression by reverse transcription (RT) -PCR, ${ }^{2}$ others reported presence in the severe psoriasis patients.

- Is there any technical issue to address this inconsistency?

- Here, we report that the difference of two method of neutrophil separation and IL-17 may not be produced from neutrophils.

\section{Material and Methods}

Flow cytometry

- Whole blood was collected from psoriasis patient and healthy control into heparinized blood collect tubes.

- Neutrophils, monocytes and lymphocytes were isolated by density gradient centrifugation with Histopaque ${ }^{\circledR}-1077$ and Histopaque ${ }^{\circledR}$ 1119.

- Neutrophil was also isolated by specific magnetic bead separation from whole blood.

- The collected cells were stained with fluorescein isothiocyanate (FITC) conjugated anti-CD14 antibody and PerCp-conjugated anti-CD3 antibody and analyzed by flow cytometry.

\section{$R T-P C R$}

- Peripheral blood mononuclear cells (PBMC) isolated by Histopaque $^{\circledR}-1077$ were purified by monocytes or $\mathrm{CD}^{+} \mathrm{T}$ cells specific magnetic bead separation.

- mRNA was extracted from collected cells by ISOGEN-LS ${ }^{\circledR}$

- The primer for RT-PCR were IL-17A and internal control glyceraldehyde 3-phosphate dehydrogenase (GAPDH) .

\section{Result}

- Neutrophils from a severe psoriasis patient purified by density gradient centrifugation showed merged zone between neutrophils, monocytes and $\mathrm{CD}^{+} \mathrm{T}$ cells (Fig. 1a).

- Neutrophils separated by specific magnetic bead separation shows high purity both healthy control and psoriasis patient (Fig. 1b).

- Neutrophil fraction purified by density gradient centrifugation expressed IL-17A mRNA amplification (lanes 2 and 6), however, no obvious band was detected in the neutrophil fraction separated by specific bead separation (lanes 3 and 7). (Fig. 1c)

\section{Conclusion}

- In severe psoriasis patients, merged zones among the neutrophils, mononuclear cell and $\mathrm{T}$ cell boundary were shown, resulting in low purity in neutrophil fraction.

- Our previous report declared activation of the circulating neutrophils and monocytes in psoriasis ${ }^{5}$, the activated neutrophils are morphologically enlarged with low density.

- The boundaries of each cell population were merged in flow cytometry, and this resulted in mixture of monocytes and lymphocytes with neutrophils. Thus, these aberrant cells may have a role in misunderstanding IL-17A production from neutrophils.

- Neutrophils are not the actual major source of IL-17A in psoriasis, but activated monocytes and lymphocytes are the dominant source of IL-17A. The mixture of monocytes or lymphocyte derived IL-17A mRNA was detected in the neutrophil fraction by RT-PCR.

\section{Reference}

1. Lin AM, et al. J Immunol 2011; 187: 490-500.

Tamarozzi $F$ et al. Immunol Lett 2014: 162: 194-198. 